\title{
Heranwachsen in der digitalen Welt: Chancen und Risiken für die Entwicklung
}

\section{Daniel Hajok}

Der Jugendmedienschutz ist in der Bundesrepublik Deutschland von jeher ein hohes Gut. Abgeleitet aus dem im Grundgesetz (GG) festgeschriebenen Recht eines jeden auf freie Entfaltung seiner Persönlichkeit (Art. 2 Abs. 1 GG) und in Verbindung mit dem verfassungsrechtlichen Schutzauftrag des Staates (Art. 1 Abs. 1 GG), ist es Ziel und Auftrag des Staates, Kinder und Jugendliche vor solchen Medien zu schützen, die sie in ihrer Entwicklung oder Erziehung zu einer eigenverantwortlichen und gemeinschaftsfähigen Persönlichkeit beeinträchtigen oder (schwer) gefährden können; es geht um die effektive Reduzierung von medieninduzierten Entwicklungsrisiken (vgl. Dreyer 2013). Nachfolgender Beitrag spannt den Bogen etwas weiter und systematisiert zunächst, dass faktisch alle Bereiche der Entwicklung junger Menschen heute auch medialen Einflüssen unterworfen sind. Welche Chancen sich Heranwachsenden für ein partizipatives Medienhandeln bieten und wie sie diese annehmen, spielt dabei ebenso eine Rolle, wie die erweiterten Risikolagen in der digitalen Welt, die mit Befunden zur Verbreitung der Gefahren auch Fokusthemen des Jugendmedienschutzes definieren.

\section{Persönlichkeitsentwicklung unter neuen Vorzeichen}

Die Kompetenzen für Handlungsfähigkeit, Eigenverantwortlichkeit und Gemeinschaftsfähigkeit bilden Kinder und Jugendliche bekanntlich in der Auseinandersetzung mit sich und ihrer Umwelt erst noch aus. Sie sind daher auch noch besonders sempfänglich für all die vielen neuen Inputs in ihrem Leben und können durch reale wie durch mediale Erfahrungen in ihrer Persönlichkeitsentwicklung positiv wie negativ beeinflusst werden. Medien >wirken dabei aber nicht per se auf die Entwicklung ein. Vielmehr eröffnet die zunehmend aktive und selbständige Aneignung von Medien im Zusammenspiel mit individuellen, sozialen und situativen Faktoren spezifische Potenziale und Risiken für das Heranwachsen. Diese lassen sich in allen Entwicklungsbereichen beobachten und ausgehend von einer Systematisierung an anderer Stelle (vgl. Hajok 2019a) wie folgt skizzieren.

\section{Kognitive und sprachliche Entwicklung}

Bereits die Ausbildung der ersten kognitiven Fähigkeiten (Wiedererkennen von Dingen, symbolisches Denken, Erkennen von
Zusammenhängen) sind vielfach medial unterstützt. Für die ersten Schritte der Aneignung von Symbolen und Bildern sind mediale Abbildungen in der direkten Umwelt der Kinder und Bilderbücher von besonderer Bedeutung. Ebenso wird die Entwicklung des begrifflichen Denkens und der Sprache schon früh in markanten Punkten medial unterstützt-durch das Vorlesen von Geschichten, das Sehen von Kindersendungen im Fernsehen und anderes mehr. Mit dem Erwerb basaler Fähigkeiten (Lesen, Schreiben, Rechnen), die eine zunehmend selbständige Aneignung der Welt ermöglichen, steigt das >Beeinflussungspotenzial von Medien spürbar an.

Auch die Entwicklung zunächst des logischen und rationalen, dann des abstrakten und hypothetischen Denkens ist vielfach medial angeregt. Im Medienumgang werden von den Heranwachsenden permanent

Dr. Daniel Hajok ist Kommunikations- und Medienwissenschaftler. Er ist Honorarprofessor an der Universität Erfurt und Gründungsmitglied der Arbeitsgemeinschaft Kindheit, Jugend und neue Medien (AKJM).

Informationen verarbeitet, Erkenntnisprozesse initiiert und Wissen angeeignet. Potenziale für die die sprachliche wie für die kognitive Entwicklung und Bildung einer seigenen Meinung ergeben sich dann in der differenzierten Auseinandersetzung medialen Inputs. Altersunangemessene, überfordernde und beängstigende Inhalte können hingegen die kognitive Entwicklung beeinträchtigen, als sgesetzt propagierte Meinungen noch im Jugendalter eine Internalisierung evozieren.

\section{Körperlich-physiologische und sexuelle Entwicklung}

Medien bieten vielfältige Anregungen bei der (möglichst ungestörten) Entwicklung hin zum körperlich und geistig gefestigten Erwachsenen. Gut gemachte und zielgruppenspezifische Aufklärungs-, Informationsund Beratungsangebote (etwa zu Ernährung und Gesundheit) können hier unterstützen, als nachahmens- und anstrebenswert dargestellte problematische Verhaltensweisen und psychopathologischen Störungen (etwa von selbstschädigendem Verhalten, Essstörungen) dagegen die körperlichphysiologische Entwicklung mittelbar schädigen. In engem Zusammenhang mit der körperlich-physiologischen Entwicklung ist auch der Bereich der sexuellen Entwicklung zu sehen. Ein Rolle spielen hier nicht nur die körperlichen Veränderungsprozesse (Ausbildung primärer Geschlechtsmerkmale, Geschlechtsreife), sondern auch die aktive Auseinandersetzung damit.

Die persönlichen Skripte von Sexualität bilden sich bereits in Kindheit und Vorpubertät aus und stehen dabei nicht nur unter dem Einfluss ihrer Beziehungserfahrungen (zunächst im nicht-sexuellen Bereich), sondern schnell auch von sexualitätsbezogenen medialen Darstellungen, mit denen Kinder in der Regel ungewollt konfrontiert werden. Jugendliche wenden sich schon (gezielt) expliziteren Darstellungen zu. Sie beginnen sich auch online sexy zu präsentieren und sind im Netz nicht selten den sexualisierten Grenzüberschreitungen anderer ausgesetzt. Sexualpädagogische Aufklärungs- und Beratungsangebote unterstützen die Entwicklung einer selbstbestimmten und gleichberechtigten Sexualität der Geschlechter. Darstellungen von sexueller Gewalt, bizarren Sexualpraktiken als üblich und normal können sexualethisch desorientieren, Grooming die adäquate sexuelle Entwicklung nachhaltig gefährden.

\section{Identitätsbildung}

Populäre klassische Medienangebote (etwa des Fernsehens) und die beliebten Social Media Angebote repräsentieren diverse Stereotype, Verhaltensweisen und (Geschlechter-)Rollen, die ein überaus wichtiger Input für die Identitätsbildung sind. Medien bieten den Heranwachsenden hier vielfältige positive (etwa i.S. von Geschlechtergerechtigkeit), aber auch negative (etwa i.S. der Verfestigung von Ungleichheiten) Anregungen. Die aktive Auseinandersetzung mit den identitätstypischen Fragen (Wer bin ich? Wer will ich sein? Als wen sehen mich die anderen?) ist für Jugendliche heute ohne Instagram, WhatsApp \& Co. eigentlich gar nicht mehr vorstellbar.

Sowohl für die Ausbildung einer (einmaligen und besonderen) persönlichen Identität als auch einer (mit anderen geteilten) sozialen Identität sind die medial vermittelten und online verhandelten Identitätsentwürfe, Wertvorstellungen, gesellschaftliche Ansprüche etc. mittlerweile existenziell. Allerdings unterliegt die Identitätsbildung in den mediatisierten Gemeinschaften mit jedem noch so kleinen Detail von Ich-Erprobung und sozialem Rückkanal den Beson- 
derheiten der digitalen Welt (Persistenz, Duplizier- und Sklierbarkeit). Das Selbst und die Reaktionen der anderen werden hier nicht nur öffentlich verhandelt, sondern auch dauerhaft konserviert, auffindbar, kopierbar, in andere Kontexte übertragbar, was die Identitätsarbeit zu einem chancenund zugleich risikoreichen Unterfangen macht (vgl. Hajok 2018, 2020).

\section{Soziale und ethisch-moralische Entwicklung}

Medial vermittelte Modelle für Erziehung, Partnerschaft, Familie etc. bieten vielfältige Möglichkeiten für soziale Vergleichsprozesse. Wichtig für die Entwicklung des Sozialverhaltens und Ausbildung der Fähigkeit zu Empathie bzw. der Fähigkeit zu Perspektivübernahme sind bereits die parasozialen Beziehungen zu den ersten Medienfiguren. Bereits am Ende der Kindheit steht die soziale Entwicklung in besonderem Maße unter dem Einfluss der Begegnungen mit realen Menschen in Messengerdiensten und Sozialen Netzwerken, die dort und in den face-to-face-Settings auch aktiv bearbeitet werden. Im Resultat kann sich - medial evoziert - ein von Respekt geprägtes Sozialverhalten ebenso entwickeln wie ein von Häme und Ausgrenzung gekennzeichnetes (eskaliertes) soziales Konfliktverhalten.

Die für die ethisch-moralische Entwicklung kennzeichnende Ausbildung eines moralischen Urteils ist in Medienaneignungsprozesse eingebettet, bei denen zunächst das Verhalten von Medienfiguren, später auch medial vermittelte politische Prozesse, Krisen- und Kriegsereignisse in das persönliche Menschen- und Weltbild eingeordnet werden. Werden $>$ unsere $<$ Werte und soziale Normen selbstverständlich von den Protagonisten vorgelebt, kann dies die Ausbildung eines Bewusstseins für Verantwortung und regelkonformes Handeln fördern. Nachteilig kann sich gestalten, wenn Identifikationsfiguren Devianz und Delinquenz als erfolgreiches Handeln propagieren und so eine aktive Aneignung abweichenden Verhaltens nahelegen.

\section{Religiöse und politische Sozialisation}

Neben Elternhaus und Schule bieten heute auch die Medien vielfältige Inputs für die Ausbildung eines individuell-reflektierenden Glaubens im Sinne einer existenziellen Sinnsuche und Sinnfindung. Den Grundfragen menschlicher Existenz (etwa nach einem Leben nach dem Tod oder einer shöheren Macht ) wird vor einem übersinnlichen, göttlichen oder transzendenten Hintergrund nachgegangen. Förderlich sind hier Angebote zur interreligiösen und interkulturellen Bildung, nachteilig propagandistische Schriften, YouTube-Videos etc. (etwa von Protagonisten eines gewaltorientierten Islam), die Allmacht und Vorherr- schaft bestimmter Glaubensrichtungen propagieren und die grundgesetzlich geschützte Religionsfreiheit konterkarieren.

Die Entwicklung zu einem Mitglied der Gesellschaft, das sich mit Akzeptanz der politischen Ordnung eigene Standpunkte erarbeitet und aktiv an der (Mit-) Gestaltung seiner sozialen und politischen Umwelt beteiligt, ist heute eng mit den Partizipationsmöglichkeiten im Social Web verschränkt. Hier bieten sich heute vielfältige Möglichkeiten eines sog. Hashtag-Aktivismus, bei dem Heranwachsende zunehmend auf niedrigschwellige, lebenswelt- und erlebnisorientierte Beteiligungsformen setzen (vgl. Eisewicht 2019) und sich so frühzeitig und in bisher nicht gekannter Weise eigenaktiv in politische Diskurse einbringen. ${ }^{1}$ Dies fördert die politische Sozialisation, mit Hate Speech und Fake News bahnen sich aber auch Grenzüberschreitungen ihren Weg, die einer adäquaten Entwicklung von Gemeinschaftsfähigkeit und politischem Bewusstsein entgegen stehen.

\section{Chancen: Möglichkeiten eines partizipativen Medienhandelns}

Eines ist soeben deutlich geworden: Neben den medieninduzierten Entwicklungsrisiken, an denen das Handeln des Jugendmedienschutzes von jeher orientiert ist, eröffnen sich Heranwachsenden gerade mit den neuen Möglichkeiten digitaler Medien auch Chancen bei der Entwicklung (und Erziehung) zu einer eigenverantwortlichen und gemeinschaftsfähigen Persönlichkeit, die sich letztlich für alle markanten Bereiche der Entwicklung junger Menschen konstatieren lassen. Im Fachdiskurs werden die Potenziale vor allem im Sinne eines partizipativen Medienhandelns gesehen (vgl. Wagner \& Würfel 2013). Zu unterscheiden sind hier vier grundlegende Dimensionen des Medienumgangs von Heranwachsenden, die sich mit aktuellen Zahlen wie folgt fundieren lassen.

\section{Information und Orientierung}

Partizipatives Medienhandeln meint erstens die neuen Formen von Information und Orientierung, mit denen sich Heranwachsende immer früher persönliche $\mathrm{Zu}$ gänge zu eigen machen, sie miteinander teilen, interessengeleitet aber auch immer ihre ganz eigenen Medienmenüs zusammenstellen. Im Mittelpunkt stehen hier subjektiv relevante Informationen, die sie als nützlich für das eigene Leben erachten, ihnen Orientierung bieten und nicht zuletzt auf die zunehmend selbständige Aneignung von (schulischem) Wissen verweisen.

Nach den Daten der aktuellen EU Kids Online-Befragung in Deutschland nutzt am Ende der Grundschulzeit bereits jede/r Zweite mindestens wöchentlich das Netz für Schularbeiten, im Weiteren geht es um die Suche nach Produkten (und ihren Preisen), Nachrichten/News und Infos zur Gesundheit (vgl. Hasebrink et al. 2019). In der letzten BITKOM-Studie ist zu lesen, dass von den 6- bis 7-jährigen Internetnutzer*innen jeweils jede/r Achte das Netz zumindest $a b$ und zu nutzt, um Infos zu persönlichen Interessen bzw. für Schule und Ausbildung zu suchen, bei den 16- bis 18-Jährigen sind es bereits die mit Abstand meisten (vgl. Berg 2019).

Insbesondere die Videoplattform YouTube, die seit vielen Jahren das beliebteste Internetangebot von Heranwachsenden schlechthin ist und mit der mobilen Anwendung den Sprung auf die mobilen Endgeräte Heranwachsender geschafft hat, ist heute eine wichtige Größe für Information und Orientierung. $\mathrm{Zu}$ verweisen ist hier zum einen auf die Tutorials und Erklärvideos für Themen aus der Schule, die jeweils jede/r fünfte Jugendliche täglich oder mehrmals pro Woche nutzt. Noch beliebter sind zum anderen die Channels der beliebten YouTuber*innen, die Einblick in ihren Alltag geben oder Videos über aktuelle Nachrichten präsentieren (vgl. MPFS 2018).

\section{Austausch und Vernetzung}

Einen besonderen Stellenwert für ein partizipatives Medienhandeln Heranwachsender haben zweitens die Möglichkeiten zu kommunikativem Austausch und Vernetzung, die mit dem eigenen Smartphone in der Hand längst alltagsrelevant sind: Mit zehn Jahren nutzen die meisten bereits aktiv WhatsApp, mit 14 Jahren auch Instagram und Snapchat (vgl. Berg 2019). Mit den Diensten etablieren die Heranwachsenden schnell seigene Handlungs- und Erfahrungsräume, in denen sie sich weitgehend unbehelligt von Erziehenden und pädagogischen Fachkräften zusammenfinden und austauschen können. Das unterstützt den notwendigen Umbau der sozialen Beziehung weg von den Eltern hin zu den Peers und ermöglicht zudem den Erwerb von (mediatisierter) Sozialkompetenz.

Das Soziale, was in den letzten Jahren immer weniger in Settings physischen Beisammenseins erfolgte (vgl. MPFS 2018 2019), ist mit den neuen Formen von Austausch und Vernetzung nicht verschwunden. Es ist nur immer mehr ins Netz gewandert. Im Mittelpunkt stehen hier früh die Sozialen Netzwerke, Instagram seit einigen Jahren mehr als Facebook. Für den Austausch mit Freunden und Familie sind neben WhatsApp für nicht wenige auch Skype bzw. Facetime relevant. Im Altersverlauf erweitern die Heranwachsenden dann ihre Zugänge und nehmen zunehmend auch an Austauschformen außerhalb der eigenen Peergroup teil. Im Alter zwischen 15 und 17 sind schon zwei von fünf Jugendli- 
chen regelmäßig in einer Onlinegruppe mit Menschen gleicher Interessen und Hobbys aktiv (vgl. Hasebrink et al. 2019).

Richtet man den Blick auf die beliebtesten Dienste, dann zeigt sich zweierlei: Zum einen ist WhatsApp längst auch für schulische Belange alltagsrelevant. Mittlerweile ist knapp die Hälfte der 6- bis 13-Jährigen Teil eines Klassenchats bzw. in einer Gruppe für die eigene Schulklasse unterwegs (vgl. MPFS 2019). Zum anderen ist der Austausch bei Instagram vor allem von einer (eher passiven) Teilhabe an dem, was andere (Bekannte, Stars und Prominente) dort posten, gekennzeichnet. Aktiv partizipieren Jugendliche dort vor allem durch das Kommentieren der Fotos und Videos anderer, weniger durch das Einstellen eigener Inhalte (vgl. MPFS 2018).

\section{Artikulation und Selbstausdruck}

Die soeben angesprochene aktive Teilhabe verweist bereits auf die dritte Dimension eines partizipativen Medienhandeln. Hier geht es um die Frage, inwieweit sich Heranwachsende der produktiven und kreativen Formen zu Artikulation und Selbstausdruck bedienen und die digitale Welt mit eigenen Texten, Bildern, Videos etc. bereichern. Auf diese Weise können junge Menschen heute auch das erfahren, worauf in pädagogischen und erzieherischen Kontexten vielfach abgestellt wird: Selbstwirksamkeit. Hier geht es nicht zuletzt um die wichtige Erfahrung, der sozialen und dinglich-materiellen Umwelt nicht nur passiv gegenüber zu stehen, sondern selbst Einfluss auf die Dinge zu nehmen und die (digitale) Welt aktiv mitzugestalten.

Mit eigenen Foren und Blogs oder YouTube-Videos haben in der Vergangenheit eher wenige Heranwachsende diese Möglichkeiten wahrgenommen. Nach den Daten der JIM-Studien stellten in den letzten Jahren nur deutlich unter fünf Prozent der 12- bis 17-Jährigen eigene Videos auf YouTube ein. In den (teil-)öffentlichen Vernetzungen der populären Austauschdienste sieht das etwas anders aus. Deutlich verbreiteter sind Artikulation und Selbstausdruck bereits bei den Nutzer*innen von Instagram. Hier postet jede/r Achte »häufig « eigene Fotos bzw. Stories. In den begrenzten Kommunikationsräumen von WhatsApp und Snapchat agieren die meisten Heranwachsenden regelmäßig nicht nur rezeptiv, sondern auch produktiv: Die meisten artikulieren sich in den Chats und Gruppen mit Texten, Bildern und Sprachnachrichten bzw. verschicken selbst Snaps (vgl. MPFS 2018).

\section{Kooperation und Kollaboration}

In engen Zusammenhang mit den neuen Möglichkeiten zu Austausch und Vernetzung ist auch die vierte Dimension partizi- pativen Medienhandelns zu sehen. Denn im Social Web etablieren Heranwachsende auch verschiedene Formen der Zusammenarbeit und `bearbeiten $>$ dabei gemeinschaftlich mediale Inhalte und Strukturen - und erschaffen dabei auch neue. Das Spektrum reicht hier vom Taggen, Liken oder Kommentieren dessen, was andere im Netz posten, bis hin zum Einstellen eigener Inhalte, insbesondere wenn sie damit aktiv ihre seigene Expertise in öffentliche Diskurse einbringen oder sich an kollaborativen NetzProjekten beteiligen.

Solche Produsage-Prozesse, bei denen sich Nutzung und gemeinschaftliche Produktion miteinander verschränken (vgl. Bruns 2009), haben gerade dann ein hohes partizipatives Potenzial, wenn sie selbstbestimmt und selbstverwaltet verlaufen. Faktisch müssen sie aber kommerziellen Strukturen folgen. Die sich im Social Web konstituierte Fridays for Future-Bewegung, bei denen sich Heranwachsende die Möglichkeiten des Hashtagaktivismus zu eigen gemacht und den Protest dann gemeinschaftlich auf die Straße getragen haben, ist hier nach wie vor das beste Beispiel.

Empirisch belegen lässt sich, dass solche kooperativen und kollaborativen Prozesse, die über die Verhandlung des eigen Ichs hinaus gehen, erst im Verlauf des Jugendalters relevant werden. Nach den Daten der EU Kids Online-Befragung in Deutschland hatte im Jahr 2019 von den 15- bis 17-Jährigen im letzten Monat jede/r Sechste mindestens einmal pro Woche online mit anderen über politische bzw. soziale Probleme diskutiert und jede/r Zwölfte sich online an einer Kampagne oder einem Protest beteiligt oder eine Online-Petition unterschrieben (vgl. Hasebrink et al. 2019).

\section{Gefahren: Erweiterte Risikolagen in der digitalen Welt}

Wenn Jugendschützer*innen den Medienumgang von Heranwachsenden in den Blick nehmen, dann richten sie ihr Augenmerk nicht auf die Chancen, sondern auf die Risiken für die Entwicklung junger Menschen. Der Fokus liegt noch immer auf den Gefahren, die aus der Nutzung bestimmter Inhalte für die verschiedenen Altersgruppen als ungeeignet oder gar gefährlich eingestufter Inhalte resultieren. Auch bei Erziehenden und pädagogischen Fachkräften regiert in aller Regel ein risiko-fokussierter Blick. Während die Heranwachsenden selbst heute weitgehend unbefangen im Netz agieren, sorgen sich ihre Eltern vor allem um unerwünschte Online-Kontakte ihrer Schützlinge mit nicht vertrauenswürdigen Personen (vgl. Brüggen et al. 2017). ${ }^{2}$ Bei den pädagogischen Fach- und Lehrkräften stehen wiederum die inhaltsbezogenen Risiken klar im Mittelpunkt (vgl. Gebel et al. 2018).
Tatsächlich haben sich die Risiken mit den immer früheren Zugängen junger Menschen zu den onlinefähigen Endgeräten auf der einen Seite und zu Internet, Onlinediensten und Apps auf der anderen stark erweitert. Eine Ende letzten Jahres veröffentlichte Gefährdungserhebung weist nicht weniger als 35 mehr oder minder voneinander abgrenzbare Medienphänomene mit je spezifischen Gefahrenpotenzial aus (vgl. Brüggen et al. 2019). In der systematischen Betrachtung, bei der die Gefahren an den veränderten Rollen Heranwachsender beim heutigen Medienumgang festgemacht werden, lassen sich neben den inhaltsbezogenen auch (relativ neue) konsum-, kommunikations- und verhaltensbezogenen Risiken konstatieren (vgl. Dreyer et al. 2013) und mit aktuellen Daten wie folgt untermauern.

\section{Inhaltsbezogene Risiken}

Als Rezipient*innen sind Heranwachsende weiterhin Nutzer*innen vorgefertigter bzw. standardisierter Medieninhalte. Aufgrund der immer früheren, weitgehend autonom genutzten, freien Zugänge ins Netz haben sie nun aber so früh wie nie zuvor Zugang zu potenziell (schwer) jugendgefährdenden oder entwicklungsbeeinträchtigenden Inhalten und es werden bereits Kinder in bisher nicht gekanntem Ausmaß mit altersunangemessenen Inhalten konfrontiert (vgl. Hajok 2019a). Im Mittelpunkt stehen hier weiterhin (explizite) Darstellungen von Gewalt und Sexualität, die mit den Entwicklungen in der Welt digitaler Medien nicht nur neue Verbreitungswege gefunden haben, sondern auch neue Wege in die Lebenswelten junger Menschen.

Die Daten der KIM-Studien weisen seit Jahren darauf hin, dass bereits die ersten Onlinezugänge von Kindern nicht unbeschwert sind, weil hier nicht wenige auch auf ungeeignete oder als unangenehm und ängstigend empfundene Sachen stoßen. Während der Kontakt mit problematischen Fernsehinhalten immer seltener eine Rolle spielt, haben von den 6- bis 7-jährigen Internetnutzer*innen bereits fünf Prozent im Netz Kontakt mit ungeeigneten Inhalten, bei den 12- bis 13-Jährigen sind es bereits 14 Prozent. Im Kern geht es hier bereits um Darstellungen aus dem Bereich »Erotik/Pornografie« sowie um »Gewalt/Prügelszenen« und »Horror-/Gruselvideos« (vgl. MPFS 2019). Am Beispiel der wenig später bereits etablierten (gezielten) Pornografienutzung zeigt sich dann am deutlichsten, dass schon zu Beginn des Jugendalters die meisten Inhalte nutzen, die im gesetzlichen Jugendmedienschutz als schwer jugendgefährdend eingestuft werden (vgl. Bauder \& Hajok 2019).

Die Ergebnisse der aktuellen EU Kids Online-Befragung in Deutschland belegen 
nicht nur, dass die inhaltsbezogenen Risiken im Altersverlauf der jungen Nutzer*innen spürbar an Bedeutung gewinnen, sie konstatieren auch eine im Jugendalter stark zunehmende Relevanz problematischer nutzergenerierte Inhalte (Darstellungen von selbstschädigendem Verhalten und von realen Gewalthandlungen gegen Menschen und Tiere). Im Weiteren ist hier zu lesen, dass fast doppelt so viele Mädchen wie Jungen online mit belastenden, schlimmen, ängstigenden etc. Inhalten konfrontiert werden, entsprechende Erfahrungen allerdings nicht permanent, sondern von einer großen Mehrheit der 9- bis 17-Jährigen (76 Prozent) im letzten Jahr nur »ein paar Mal« gemacht wurden (vgl. Hasebrink et al. 2019).

\section{Konsumbezogene Risiken}

Als zunehmend eigenaktive Konsument*innen bzw. Marktteilnehmer*innen sind Heranwachsende heute frühzeitig Vertragspartner*innen von Medienanbietenden, die ihre Angebote und Dienste zuvorderst unter kommerziellen Gesichtspunkten ausgestalten und gezielt an die jungen Zielgruppen adressieren. In der Konsequenz stehen Heranwachsende heute vertragsrechtlichen, datenschutzrelevanten und kostenbezogenen Problemen gegenüber, die eine unbeschwerte Teilhabe (an der Online-Kommunikation) gefährden können (vgl. Brüggen et al. 2019). Das Spektrum reicht hier von unterschiedlichen >offiziellen • Altersangaben für die präferierten Dienste ${ }^{3}$ über eine intransparente Speicherung und Weitergabe persönlicher Daten (u.a. für die zielgruppenspezifische Aussteuerung von Werbung bzw. Targeting) bis hin zu undurchsichtigen Werbeformen (Product Placement, virales Marketing) und sversteckten Kosten in den beliebten Spiele-Apps.

Risiken, die den Datenschutz und die Privatsphäre der jungen Marktteilnehmer*innen betreffen, spielen in der Eigenwahrnehmung und persönlichen Erfahrung allerdings keine allzu große Rolle. Am häufigsten erleben die Heranwachsenden hier das Problem von Viren und Spyware auf ihrem digitalen Gerät: Sieben Prozent der 9- bis 17-Jährigen berichten von entsprechenden Erfahrungen innerhalb der letzten zwölf Monate. Noch weniger haben in dieser Zeit negative Erfahrungen mit der unerwünschten Nutzung ihrer Daten durch Dritte, ${ }^{4}$ der Ortung ihres Smartphones oder dem Missbrauch des eigenen Passworts gemacht. Der Anteil derjenigen, die durch Betrug im Internet Geld verloren haben, ist nach den Daten der EU Kids Online-Befragung sogar verschwindend gering (vgl. Hasebrink et al. 2019).

Mehr Relevanz zumindest als potenzielle Gefahr haben finanzielle (Vertrags-)Risiken. Das Spektrum reicht hier von Micro-
Payments und In-App-Käufen bis hin zu Gewinnspielen und unbeabsichtigten Abos. In der ersten Ausgabe des Jugendmedienschutzindex sorgte sich noch jede/r vierte der 9- bis 16-Jährigen darum, online Opfer von Kostenfallen, Abzocke oder Betrug zu werden, und jede/r Siebte gab an, dies selbst schon einmal erlebt zu haben (vgl. Brüggen 2017). Auch sah sich hier nur eine Minderheit überhaupt in der Lage, die (tatsächlich verursachten) Kosten einer App nachzuvollziehen zu können, was gerade bei den digitalen Spielen eine wichtige Größe ist. Es kann daher nicht verwundern, dass im Alter zwischen 12 und 15 Jahren jede/r Zehnte beim Spielen schon einmal versehentlich etwas gekauft oder abonniert hat-vor allem Jungen und niedriger Gebildete machen offenbar entsprechende Erfahrungen (vgl. MPFS 2018).

\section{Kommunikationsbezogene Risiken}

Als Kommunizierende nehmen Heranwachsende heute immer früher und umfangreicher an mediatisierten Austauschund Kommunikationsprozessen teil. Häufig machen sie Erfahrungen mit Gruppendruck und online ausgetragenen Konflikten, keineswegs selten mit Cybermobbing, Beleidigungen, Lügen über die eigenen Person, Hass und Hetze sowie sexualisierten Grenzverletzungen bis hin zum Grooming. Neben den vieldiskutierten Hasskommentaren, mit denen mittlerweile fast jede/r vierte Jugendliche Bekanntschaft macht (allem voran bei YouTube und Instagram) (vgl. Hasebrink et al. 2019, MPFS 2018), scheint Cybermobbing zumindest für die weiblichen Heranwachsenden das Hauptrisiko in der Onlinewelt zu sein.

Nimmt man aktuelle Studien zur Hand, dann sind beim kommunikativen Austausch im Netz zwischen zehn und 20 Prozent der ab 12-Jährigen schon einmal selbst beleidigt oder gemobbt worden bzw. wurden falsche oder beleidigende Inhalte über sie verbreitet (vgl. Berg 2019, MPFS 2018). Die als sehr belastend wahrgenommenen Mobbingerfahrungen, ${ }^{5}$ die Mädchen signifikant häufiger erleben müssen als Jungen, gehen in den meisten Fällen auf die Zeit in Klasse 5 bis 7 zurück. Die Täter*innen sind meist im näheren Umfeld der Heranwachsenden zu suchen (eigene oder andere Klasse an der Schule) (vgl. Hajok et al. 2019). Die sozialen bzw. kommunikativen Eskalationen tragen auch entscheidend dazu bei, dass die unliebsamen Erfahrungen im Netz zunehmen. Legt man die Zahlen der letzten beiden BITKOM-Studien nebeneinander, dann ist der Anteil der 10- bis 18-Jährigen mit negativen Online-Erfahrungen in nur zwei Jahren von 29 auf 41 Prozent gestiegen (vgl. Berg 2017, 2019).

Zugenommen haben in den letzten Jahren offenbar auch die Erfahrungen Heran- wachsender mit unerwünschter sexualisierter Online-Kommunikation. Im schlimmsten Fall geht es hier um das strafbewehrte Grooming, bei dem Erwachsene gezielt einen Missbrauch anzubahnen versuchen. Nach aktuellen Befragungsdaten ist - und das alles andere als gewollt - mittlerweile schon fast jede/r dritte Jugendliche im Alter zwischen 12 und 17 Jahren mehrfach im Netz nach »sexuellen Dingen « (vgl. Hasebrink et al. 2019) bzw. »schon mal nach einem Nacktbild « von sich gefragt worden, was zumeist von Unbekannten und häufig von Erwachsenen ausging (vgl. Hajok et al. 2019). Abgesehen davon zeigen die Studien, dass entsprechende Erfahrungen im Altersverlauf der Jugendlichen zunehmen und vor allem Mädchen betroffen sind.

\section{Verhaltensbezogene Risiken}

Als Produzierende bzw. Akteur*innen, die sich aktiv der Möglichkeiten digitaler Medien bedienen, sind es die Heranwachsenden zuweilen auch selbst, die bei ihrem (auf andere bezogenen) Medienhandeln die Grenzen überschreiten oder (für sich selbst) riskante bzw. dysfunktionale Medienumgangsweisen etablieren. Nicht wenige produzieren und verbreiten ungeeignete Inhalte im Netz, propagieren problematische Verhaltensweisen, attackieren andere bei der Onlinekommunikation, präsentieren sich in ihren Profilen zu freizügig, etablieren beim Sexting einen riskanten Austausch oder tauchen zu tief in die Medienwelten ein. Am Beispiel von Cybermobbing und (unerwünschter) sexualisierter Kommunikation zeigt sich sehr deutlich, dass sich innerhalb der kommunikationsbezogenen Risiken (für die Adressaten medialer Botschaften) häufig auch spezifische verhaltensbezogene Risiken (für die Absender medialer Botschaften) auftun.

Direkt auf die unrühmliche Akteursrolle angesprochen, gaben in der bereits zitierten Studie aus dem Emsland vier Prozent der befragten Schüler*innen aus den Klassenstufen 5 bis 10 offen zu, andere selbst schon einmal über eine längere Zeit hinweg online gemobbt zu haben. Mit zunehmendem Alter der Befragten hat zudem auch das Verschicken oder Posten von eigenen freizügigen Bildern und Videos eine immer größere Verbreitung (Anstieg von fünf auf 17 Prozent) (vgl. Hajok et al. 2019). ${ }^{6}$ Dass es sich hier keineswegs um ein seltenes Verhalten handelt, was mit zunehmenden Alter der Heranwachsenden an Relevanz gewinnt, zeigt auch die EU Kids OnlineBefragung in Deutschland: Hier gab jeder vierte Junge und jedes sechste Mädchen im Alter zwischen 12 und 17 Jahren an, in den letzten zwölf Monaten auch Nachrichten mit sexuellem Inhalt versendet oder gepostet zu haben (vgl. Hasebrink et al. 2019). 
Auch im Fall des einvernehmlichen individualisierten Austauschs erotischen Bildmaterials unter Jugendlichen (Sexting), der hierzulande kein verbotenes, sondern ein von der sexuellen Mündigkeit sgedecktes< Verhalten ist, bleibt der Austausch vor dem Hintergrund des nicht abwägbaren Handelns anderer stark risikobehaftet. Die Grenzen eines tolerierbaren Verhaltens werden spätestens dann überschritten, wenn das Bildmaterial weiterverbreitet oder sogar öffentlich gepostet wird (vgl. Hajok 2019c). Hierzu fehlen aber belastbare Daten.

Anders bei dem letzten hier kurz thematisierten verhaltensbezogenen Risiko: Medienabhängigkeit. Hier liegen mittlerweile mehrere ernstzunehmende Studien vor, die festgemacht an klassischen Suchtkriterien< (Kontrollverlust, Regulation negativer Gefühle, Entzugserscheinungen etc.) zwischen fünf und sieben Prozent >Medienabhängige unter Jugendlichen und weitere 20 Prozent `Gefährdete identifizieren. Die `Generation Smartphone ist damit aktuell die Hauptrisikogruppe einer Medienabhängigkeit, die sich bei Mädchen vor allem im Bereich Social Media beobachten lässt, bei Jungen im Bereich der Computerspiele (vgl. Hajok \& Seiß 2018, Hajok 2019d).

\section{Fazit}

Die Entwicklung und Erziehung junger Menschen zu einer eigenverantwortlichen und gemeinschaftsfähigen Persönlichkeit steht heute in besonderem Maße unter dem Eindruck von Medien - mit Chancen und Risiken zugleich. In der aktiven Aneignung insbesondere digitaler Medien bieten sich den Heranwachsenden heute viele Potenziale eines partizipativen Medienhandelns. Die neuen Möglichkeiten zu Information und Orientierung, Austausch und Vernetzung nutzen Jugendliche längst selbstverständlich. Nur die wenigsten bedienen sich aber regelmäßig der neuen Formen von Artikulation und Selbstausdruck, Kooperation und Kollaboration, die abseits des peergroupbezogenen Austauschs bei WhatsApp, Instagram \& Co. auf eine aktive Mitgestaltung an gesamtgesellschaftlichen Prozessen und politische Partizipation verweisen.

Mit den Chancen haben sich in der digitalen Welt aber auch die Gefahren stark erweitert. Obschon im gesetzlichen Jugendmedienschutz weiterhin die inhaltlichen Risiken des Medienumgangs im Fokus stehen, sind in der Selbstwahrnehmung wie in der Verbreitung bei der jungen Generation heute die kommunikations- und verhaltensbezogenen Risiken das dringlichste Problem - und hierauf haben die klassischen, an inhaltlichen Risiken orientierten Instrumente des gesetzlichen Jugendmedienschutzes keine adäquate Antwort. Zu fordern ist ein weiteres Mal auch hinsicht- lich des Medienumgangs eine Stärkung eines erzieherischen Jugendschutzes, wie er im SGB VIII schon längst verankert ist und nicht zuletzt in den aus der UN-Kinderrechtskonvention abgeleiteten, erstarkten digitalen Rechten von Kindern und Jugendlichen weiter ausdifferenziert ist.

1 Bestes Beispiel hier die Fridays-for-FutureBewegung, mit der Schüler*innen Entwicklungen angestoßen haben, die ein bislang nicht gekanntes Potenzial zur politischen Partizipation junger Menschen sichtbar machen, von dem auch die offizielle Politik nicht unbeeinflusst blieb.

2 Eltern von Mädchen sind hier besonders sensibel. Die Sorge, dass die eigene Tochter von Fremden im Internet kontaktiert wird, treibt mittlerweile mehr Eltern um, als die Sorge, dass ihr Kind Opfer eines Verbrechens oder auf der Straße verletzt wird (vgl. Hasebrink et al. 2019).

3 Das Mindestalter für die Nutzung von WhatsApp liegt laut AGBs bei 16 Jahren, im App Store ist der kostenlose Dienst mit »12+« und im Play Store mit »ab 0 « gelabelt. Auch YouTube sieht in den AGBs ein Mindestalter von 16 Jahren vor, ist in den Stores von Apple und Google aber mit »17+«bzw. »ab 12 « zu haben.

4 In diesem Kontext ist auch auf das sog. Sharenting zu verweisen, von dem vor allem Kinder betroffen sind. Im Alter zwischen 9 und 11 Jahren hat jede/r Zehnte bereits die Erfahrung gemacht, dass die eigenen Eltern oder Betreuer*innen Dinge (Texte, Bilder, Videos) über sie veröffentlicht haben, ohne sie vorher nach ihrem Einverständnis zu fragen.

5 In einer groß angelegten Online-Befragung von Schüler*innen der Klassenstufe 5 bis 10 im Emsland gab jede/r Dritte mit eigener Mobbingerfahrung an, (sehr) verletzt gewesen zu sein, jede/r Vierte hatte Selbstmordgedanken und jede/r Fünfte war nach eigenen Angaben verzweifelt. Die Bereitschaft, die eigenen Mobbingerfahrungen publik zu machen, ist dennoch eher gering ausgeprägt (vgl. Hajok et al. 2019).

6 Nach Angaben der Mädchen handelte es sich hier überwiegend um das Verschicken von Bildern oder Videos, in denen sie in Bikini oder Unterwäsche abgebildet sind. Die Jungen präsentieren sich demgegenüber häufiger vollständig nackt oder mit entblößtem Oberteil.

\section{Literatur}

Bauder, N. \& Hajok, D. (2019): Jugend und Pornografie. Aktuelle Perspektiven auf einen $>$ Klassiker des Jugendmedienschutzes. In: JMSReport, Jg. 42, Heft 2/19, S. 2-7.

Berg, A. (2019): Kinder und Jugendliche in der digitalen Welt. Berlin.

Berg, A. (2017). Kinder und Jugend in der digitalen Welt. Berlin.

Brüggen, N./Dreyer, S. /Drosselmeier, M./Gebel, C./ Hasebrink, U. / Rechlitz, M. (2017): Jugendmedienschutzindex: Der Umgang mit onlinebezogenen Risiken - Ergebnisse der Befragung von Eltern und Heranwachsenden. Berlin.

Brüggen, N. / Dreyer, S. / Gebel, C. / Lauber, A. / Müller, R. / Stecher, S. (2019): Gefährdungsatlas. Digitales Aufwachsen. Vom Kind aus denken. Zukunftssicher handeln. Bonn.
Bruns, A. (2009): Produtzung: Von medialer zu politischer Partizipation. In: C. Bieber / M. Eifert / Th. Groß / J. Lamla (Hrsg.), Soziale Netze in der digitalen Welt. Das Internet zwischen egalitärer Teilhabe und ökonomischer Macht. Frankfurt a.M., S. 65-86.

Dreyer, S. (2013): Rechtliche Grundlagen des Jugendmedienschutzes. In: H. Friedrichs / T. Junge / U. Sander (Hrsg.): Jugendmedienschutz in Deutschland. Wiesbaden, S. 65-82.

Dreyer,S./Hasebrink,U./Lampert, C./Schröder,H.-D. (2013): Herausforderungen für den Jugendmedienschutz durch digitale Medienumgebungen. In: Soziale Sicherheit (CHSS), Heft 4/2013, S. 195-199.

Eisewicht, P. (2019): Zwischen Straßenbarrikade und Hashtagaktivismus. Zum Wandel des Engagements Jugendlicher in der modernen Gesellschaft. In: Thema Jugend, Heft 2-2019, S. 6-8.

Gebel, C. / Brüggen, N. / Hasebrink, U. /Lauber, A. / Dreyer, S. / Drosselmeier, M. / Rechlitz, M. (2018): Jugendmedienschutzindex: Der Umgang mit onlinebezogenen Risiken. Ergebnisse der Befragung von Lehrkräften und pädagogischen Fachkräften. Berlin.

Hajok, D. (2020): Alles anders? Wie sich Jugend in der digitalen Welt gewandelt hat. In: deutsche jugend, Jg. 68, Heft 1, S. 11-18.

Hajok, D. (2019a): Heranwachsen in der zunehmend mediatisierten Gesellschaft: Kinder und Jugendliche im Spannungsfeld digitaler Medien. TPJ - Theorie und Praxis der Jugendhilfe, Heft 24, 8-35.

Hajok, D. (2019b): Verändertes Heranwachsen mit den Risiken der Onlinewelt. Fakten und Möglichkeiten von Prävention. In: ZJJ - Zeitschrift für Jugendkriminalrecht und Jugendhilfe, Jg. 30, Heft 4, S. 367-374.

Hajok, D. (2019c): Sexting in digitalen Medien. Riskantes Austauschhandeln Jugendlicher im Fokus. In: DVJJ (Hrsg.), Herein, Heraus, Heran - Junge Menschen wachsen lassen. Mönchengladbach, S. 415-432.

Hajok, D. (2019d): Tauchen Jugendliche zu tief in die Welt digitaler Medien ein? In: tv diskurs - Verantwortung in audiovisuellen Medien, Jg. 23, Heft 1, S. 13-15.

Hajok, D. (2018): Digitale Kommunikation: Wie sozial ist social media? In: THEMA JUGEND, Heft 4-2018, S. 8-10.

Hajok, D. \& Seiß, L. (2018): Sucht, Abhängigkeit? Oder doch nur ein ganz normales Heranwachsen mit digitalen Medien? In: JMSReport, Jg. 41, Heft 5/18, S. 6-10.

Hajok, D. / Siebert, P. / Engling, U. (2019): Digital $\mathrm{Na}(\mathrm{t})$ ives. Ergebnisse der Wiederholungsbefragung und Konsequenzen für den präventiven Jugendmedienschutz. In: JMS-Report, Jg. 42, Heft 1/19, 2-5.

Hasebrink, U./Lampert, C./Thiel,K.(2019): OnlineErfahrungen von 9- bis 17-Jährigen. Ergebnisse der EU Kids Online-Befragung in Deutschland 2019. Hamburg.

MPFS (Medienpädagogischer Forschungsverbund Südwest) (Hrsg.) (2019): KIM-Studie 2018. Basisuntersuchung zum Medienumgang 6- bis 13-Jähriger in Deutschland. Stuttgart.

MPFS (Medienpädagogischer Forschungsverbund Südwest) (Hrsg.) (2018): JIM-Studie 2018. Basisuntersuchung zum Medienumgang 12bis 19-Jähriger. Stuttgart.

Wagner, U. \& Würfel, M. (2013): Gesellschaftliche Handlungsfähigkeit in mediatisierten Räumen. In A. Hartung / A. Lauber / W. Reißmann (Hrsg.), Das handelnde Subjekt und die Medienpädagogik. München, S. 159-167. 